\title{
The power of food science and technology and nutrition for sustainable planet health: food processing saves lives
}

\author{
Fereidoon Shahidi
}

\begin{abstract}
Department of Biochemistry, Memorial University of Newfoundland, St. John’s, NL. Canada A1C 5S7. E-mail: fshahidi@mun.ca
\end{abstract} DOI: $10.31665 / J F B .2021 .16286$

Received: December 21, 2021; Revised received \& accepted: December 25, 2021

Citation: Shahidi, F. (2021). The power of food science and technology and nutrition for sustainable planet health: food processing saves lives. J. Food Bioact. 16: 1-2.

Food processing includes a chain of events that starts in the farm and continues to the serving plate. These may include washing, dehydration, blanching, heat processing, and packaging, among others. While processing is essential for providing a safe food supply by eliminating harmful microorganisms and certain undesirable components, as well as revealing the desirable flavor of food, there has been much recent attempt to condemn food processing. Thus, loss of certain nutrients during processing and use of additives in ready to eat food as well as high levels of salt, sugar and fat/oil have been noted as being responsible for many noncommunicable diseases due to increased incidence of obesity, diabetes and cardiovascular disease. These have all been categorised as ultra-processed food but attention to the definitions and details is most important.

To provide a balanced and scientifically based view, a twoday food conference was held on November 16 and 17, 2021 under the auspices of International Science Council (ISC) by the International Union of Food Science and Technology (IUFoST) and the International Union of Nutritional Sciences (IUNS). The conference discussed many issues related to food sustainability and safety in order to "cleaning up the confusion and controversy about processed food and health". Of the several sessions in the conference, the one on Innovations in Food Science and Technology for Sustainable Food Processing is of special interest and hence this brief focusses on the aspects of Food Processing Saves Lives to highlight the points. Professors Susan Socolovsky of the University of Buenos Aires and Roger Clemens of the University of Southern California provided an excellent recount of the shortcomings of the definitions and details. Portraying all processing as ultra-processing and equating them with junk food is an over-simplification. For example, while ultra-high temperature (UHT) milk is considered OK, but adding chocolate makes it ultra-processed. In addition, while excess lipid is detrimental to health, certain segment of the society may need that as a source of high calorie food. Similarly, while high content of salt in food is responsible for high blood pressure, it is needed for hot climate regions to provide electrolyte balance and to provide iodine to prevent goiter. Replacing sucrose with less desirable alternatives may also defeat the purpose as it was the case for replacing animal fat containing a high saturated fatty acid level with partially hydrogenated vegetable oil that ended up being even more harmful to health. Therefore, a diet that takes advantage of balanced inclusion of nutrients and necessary components as well as optimum processing conditions that preserves and retains the bioactive components should be recommended. These points were emphasized by the presenters or are made here by the author.

Other speakers in this session were Professors Alexander Mathys of Swiss Federal Institute of Technology and Mitsutoshi Nakajima of the University of Tsukuba who spoke about "Novel food production and diets for more sustainable food systems" and sustainable solutions through disruptive technologies", respectively. This first session was chaired by Professor Aman Wirakartakusumah (Presiding Officer of the IAFoST) and Professor Fereidoon Shahidi (Chair of the Scientific Council of IUFoST).

The second session of the conference was on Integrating Capacity Building and Future of Food Science, chaired by Professor Cristina Silva of the Portuguese Catholic University and Dr. Sebastiano Porretta of the Italian Ministry of Economic Development. The speakers of this sessions were Professor Sam Saguy of the Hebrew University of Jerusalem who talked about the Future of Food Science, Dr. Ferruh Erdogdu of Ankara University who elaborated on Engineering Food Science and Dr. Azis Boing Sitanggang of IPB University who spoke about Future Opportunities for Young Food Scientists.

The first day sessions were concluded with a lengthy discussion and question and answer period, and this was chaired by Dr. Vish Prakash (President of IUFoST) and Dr. Alfredo Martinez (president of IUNS).

The second day of the conference was on Sustainable Food Safety in Processing, chaired by Dr. Pingfan Rao, Professor and Director of the Chinese Academy of Science Shanghai Institutes of Biological Sciences-Zhejiang Ghongshang University Joint Centre of Food and Nutrition Research, and Dr. Hongda Chen of the US Department of Agriculture. The speakers in this session were Dr. Samuel Godefroy of Universite Laval who spoke about Steering Food Safety through Regulatory Processes, Dr. Ratih Dewanti-Hariyadi, ICMSF Board and IPB University presenting on Developing Microbiological Criteria to Improve Food Safety: 
Lessons Learned from Indonesia. This was followed by a presentation from Professor Charles Aworh of the University of Ibadan speaking about the Traditional Food Processing and Safety and finally a presentation by Professor Carol Ann Wallace of the University of Central Lancashire who talked about the Food Safety Culture, an Essential Ingredient for Safe and Healthy Diet. The session was again concluded by a Discussion period chaired by Dr. Vish Prakash and Dr. Alfredo Martinez.

The conference was considered a success as it brought experts from both Food Science and Nutrition together and there is much we can learn from each other.

For further information about the conference and details, please visit the following links.

Programme and access to ppt presentations: https://council. science/events/food-science-technology-nutrition-sustainableplanet-health/.

YouTube Recording November 16: https://www.youtube.com/ watch? $\mathrm{v}=\mathrm{xYHYnM} 73 \mathrm{ocA}$.

YouTube Recording November 17: https://www.youtube.com/ watch?v=77esF_U3L-8. 